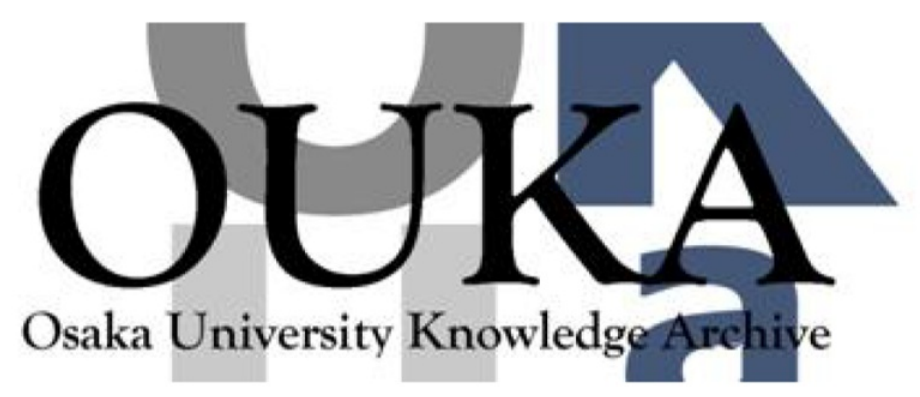

\begin{tabular}{|c|c|}
\hline Title & $\begin{array}{l}\text { Visible Fluorescence Induced by the Metal } \\
\text { Semiconductor Transition in Composites of } \\
\text { Carbon Nanotubes with Noble Metal Nanoparticles }\end{array}$ \\
\hline Author (s) & $\begin{array}{l}\text { Subramaniam, Chandramouli; Sreeprasad, T.S; } \\
\text { Pradeep, T et al. }\end{array}$ \\
\hline Citation & $\begin{array}{l}\text { PHYSICAL REVIEW LETTERS. 99(16) p. 167404-1- } \\
\text { p. } 167404-4\end{array}$ \\
\hline Issue Date & $2007-10-19$ \\
\hline oaire:version & VoR \\
\hline URL & https://hdl. handle. net/11094/2859 \\
\hline rights & $\begin{array}{l}\text { Subramaniam, Chandramouli, Sreeprasad, T.S, } \\
\text { Pradeep, T, Kumar, G. V. Pavan, Narayana, } \\
\text { Chandrabhas, Yajima, T, Sugawara, Y, Tanaka, } \\
\text { Hi rofumi, Ogawa, Takuji, Chakrabarti, J, } \\
\text { Physical' Review Letters, 99, 16, 167404, 2007- } \\
\text { 10-19. "Copyright } 2007 \text { by the American Physical } \\
\text { Society." }\end{array}$ \\
\hline Note & \\
\hline
\end{tabular}

Osaka University Knowledge Archive : OUKA

https://ir. Library. osaka-u. ac. jp/

Osaka University 


\title{
Visible Fluorescence Induced by the Metal Semiconductor Transition in Composites of Carbon Nanotubes with Noble Metal Nanoparticles
}

\author{
Chandramouli Subramaniam, T. S. Sreeprasad, and T. Pradeep* \\ DST Unit on Nanoscience, Department of Chemistry and Sophisticated Analytical Instrument Facility, \\ Indian Institute of Technology Madras, Chennai-600 036, India \\ G. V. Pavan Kumar and Chandrabhas Narayana \\ Light Scattering Laboratory, Chemistry and Physics of Materials Unit, Jawaharlal Nehru Centre for Advanced Scientific Research, \\ Jakkur P.O., Bangalore-560 064, India
}

\author{
T. Yajima, Y. Sugawara, Hirofumi Tanaka, and Takuji Ogawa \\ Research Center for Molecular Scale Nanoscience, Institute for Molecular Science, \\ 5-1 Higashiyama, Myodaiji, Okazaki ã 444-8787, Japan \\ J. Chakrabarti \\ S. N. Bose National Center for Basic Sciences, Sector-III, Block-JD, Salt Lake, Kolkatta-700 098, India
}

(Received 12 May 2007; published 19 October 2007)

\begin{abstract}
We show that single-walled carbon nanotube (SWNT) bundles emit visible fluorescence in the presence of noble metal nanoparticles and nanorods in the solid state. Conductivity measurements with metallic nanotubes, isolated from pristine SWNTs, show that they become semiconducting in the presence of the metal nanoparticles. Nanoparticle binding increases the defects in the nanotube structures which is evident in the Raman spectra. The metal-semiconductor transition removes the nonradiative decay channels of the excited states enabling visible fluorescence. Nanotube structures are imaged using this emission with resolution below the classical limits.
\end{abstract}

PACS numbers: 78.67.Ch, 68.37.Uv, 73.63.Fg, 78.30.Na

Isolated single-walled carbon nanotubes (SWNTs) and their composites have proved to be exciting materials from fundamental and technological perspectives. The near infrared (NIR) fluorescence [1] from isolated semiconducting SWNTs, although weak [2], has attracted a great deal of attention due to its applications in biomolecular imaging [3] and therapeutics [4]. It has not been observed in SWNT bundles due to the presence of metallic nanotubes in them, providing nonradiative decay channels [5-7]. The possibility of light emission from SWNT bundles will pave the way for novel research and applications. Here we present the first report of visible fluorescence from SWNT bundles constituting a nanotube-nanoparticle composite, with concomitant changes in the axial conductivity of SWNTs.

A nanoparticle-nanotube composite was prepared at a liquid-liquid interface. Gold and silver spherical nanoparticles ( 15 and $60 \mathrm{~nm}$ diameter, respectively) were prepared using citrate reduction [8]. Smaller gold nanoparticles of $4 \mathrm{~nm}$ mean diameter were prepared by reducing auric $\left(\mathrm{AuCl}_{4}^{-}\right)$ions using sodium borohydride [9] at $0^{\circ} \mathrm{C}$. Photochemically [10] and chemically [11] synthesized gold nanorods (AuNRs) of aspect ratios 2.8 and 3.1, respectively (15 and $12 \mathrm{~nm}$ diameter, respectively), were also used. AuNRs, preserved in a saturated solution of cetyltrimethylammonium bromide (CTAB), were cleaned by repeated sonication and centrifugation at $12000 \mathrm{~g}$. The final dispersion did not contain the protecting agent $\mathrm{CTAB}$ and was found to aggregate and precipitate within 10 minutes of redispersion. The as-prepared nanoparticles or the purified nanorods were used in the composite preparation.

SWNTs from various sources, namely, Sigma Aldrich, Carbon Nanotechnologies, Inc., and those synthesized from alcohols were used to verify the reproducibility of the results. Their average length was $\sim 20 \mu \mathrm{m}$ as reported by the supplier, although smaller lengths were detected in microscopy. SWNTs were dispersed in $N, N$-dimethyl formamide (DMF). Repeated sonication and centrifugation (at $50000 \mathrm{~g}$ ) for prolonged periods ensured that only SWNTs were present. Purified dispersion, without any surfactant, was stable for extended periods. No metallic impurities or nanoparticles were detected in the purified material. SWNTs prepared via high-pressure CO (HiPco) disproportionation route, purchased from Carbon Nanotechnologies, Inc. were used for all measurements with metallic SWNTs (mSWNTs).

Equal volumes of aqueous metal nanoparticles or nanorods (concentration, $C_{\mathrm{NP}} \approx 10^{-4} \mathrm{M}$ ) and diethyl ether were mixed to create an aqueous-organic interface. Purified SWNT dispersion in DMF (concentration, $C_{\mathrm{SWNT}} \approx 1.7 \mathrm{mg} / \mathrm{ml}$ ) was added to this biphasic system, resulting in spontaneous formation of a composite film at the aqueous-organic liquid interface. This composite was transferred to a desired substrate, after evaporation of the organic layer, for further investigations. X-ray photoelectron spectroscopy (XPS) of the composites, measured using a VG ESCA LAB MKII instrument having a $\mathrm{Mg} K \alpha$ 
source, showed no traces of $\mathrm{Fe}, \mathrm{Ni}$, or $\mathrm{Co}$, while $\mathrm{Ag} 3 d_{5 / 2}$ and $\mathrm{Au} 4 f_{7 / 2}$ were observed at $368.2 \mathrm{eV}$ and $83.8 \mathrm{eV}$, respectively. Fluorescence measurements of the concentrated $\mathrm{HCl}$ extracts of SWNTs were carried out with a FP6600 , JASCO spectrofluorimeter. A weak emission at $814 \mathrm{~nm}$, due to the cuvette, was observed. Inductively coupled plasma-mass spectrometry (ICP-MS) measurements of the extracts were done with a Shimadzu ICPS8000 atomic emission spectrometer. No traceable impurities like $\mathrm{Fe}$ and $\mathrm{Co}$ were present, while $\mathrm{Ni}(0.7 \mathrm{ppm})$ present was observed, which was due to the $\mathrm{HCl}$ used. Thus, it is clearly ascertained that no metal impurity is present in the purified SWNT sample.

Confocal Raman measurements were done with a WiTec GmbH, Alpha-SNOM CRM 200 having $514.5 \mathrm{~nm}$ argon ion laser with a $100 \times$ objective. The signal was collected in a backscattering geometry. A Peltier-cooled charge coupled device was used as the detector. An Alpha-SNOM cantilever having an aperture $(<100 \mathrm{~nm})$, with a photomultiplier detector, was used for transmission SNOM. A single spot confocal Raman spectrum of the Ag nanoparticleSWNT composite [Fig. 1(A), trace (a)] shows the characteristic features such as the radial breathing mode (RBM) and the $D$ and $G$ bands at 175,1327 , and $1577 \mathrm{~cm}^{-1}$, respectively. The RBM, measured separately at higher resolution and at still lower frequencies, shows multiple modes [inset (i) of Fig. 1(A)]. Raman bands are superimposed over a large structured emission peaking at $\sim 600$ and $650 \mathrm{~nm}$, with a shoulder at $\sim 670 \mathrm{~nm}$. All features are similar in Au nanoparticle and AuNR composites also [traces (b) and (c), respectively]. Several fine structures are also observed in the range of $6000 \mathrm{~cm}^{-1}$, in all the three composites. The $G$-band intensities of the composite compared to the pristine nanotubes show enhancements $\sim 10^{3}$. The $(n, m)$ indices of SWNTs based on RBM were $(10,10),(18,0)$, and $(13,7)$ (metallic) and $(17,0),(11,9)$, and $(12,8)$ (semiconducting). The diameter of the nanotubes was estimated be $1.4 \mathrm{~nm}$ from RBM [12]. Transmission electron microscopy (TEM) analysis of the composites at lower magnifications (100 kV) showed [Fig. 1(B)] long fibrous structures consisting of isolated nanotubes and bundles with 3 to 20 SWNTs with nanoparticles in close proximity. When investigated closely (at $300 \mathrm{kV}$ ) nanotubes of diameter $\sim 1.4 \mathrm{~nm}$, consistent with the RBM estimate, surrounded by nanoparticles or nanorods were observed.

We ascertain that the visible emission is due to the composite. A film of the parent SWNT in the aqueous phase showed no visible emission [Fig. 1(A), trace (d)]. No emission was obtained with a SWNT film prepared with boiled trisodium citrate, subjected to all processes except that there was no metal ion precursor and therefore, no nanoparticles [Fig. 1(A), trace (e)]. However, other features like RBM, $D$ band, $G$ band, and $G^{\prime}$ band, were distinctly visible in all these cases. Nanoparticles and nanorods themselves did not show these features, as can be seen from the spectrum of a drop-cast AuNR film [inset
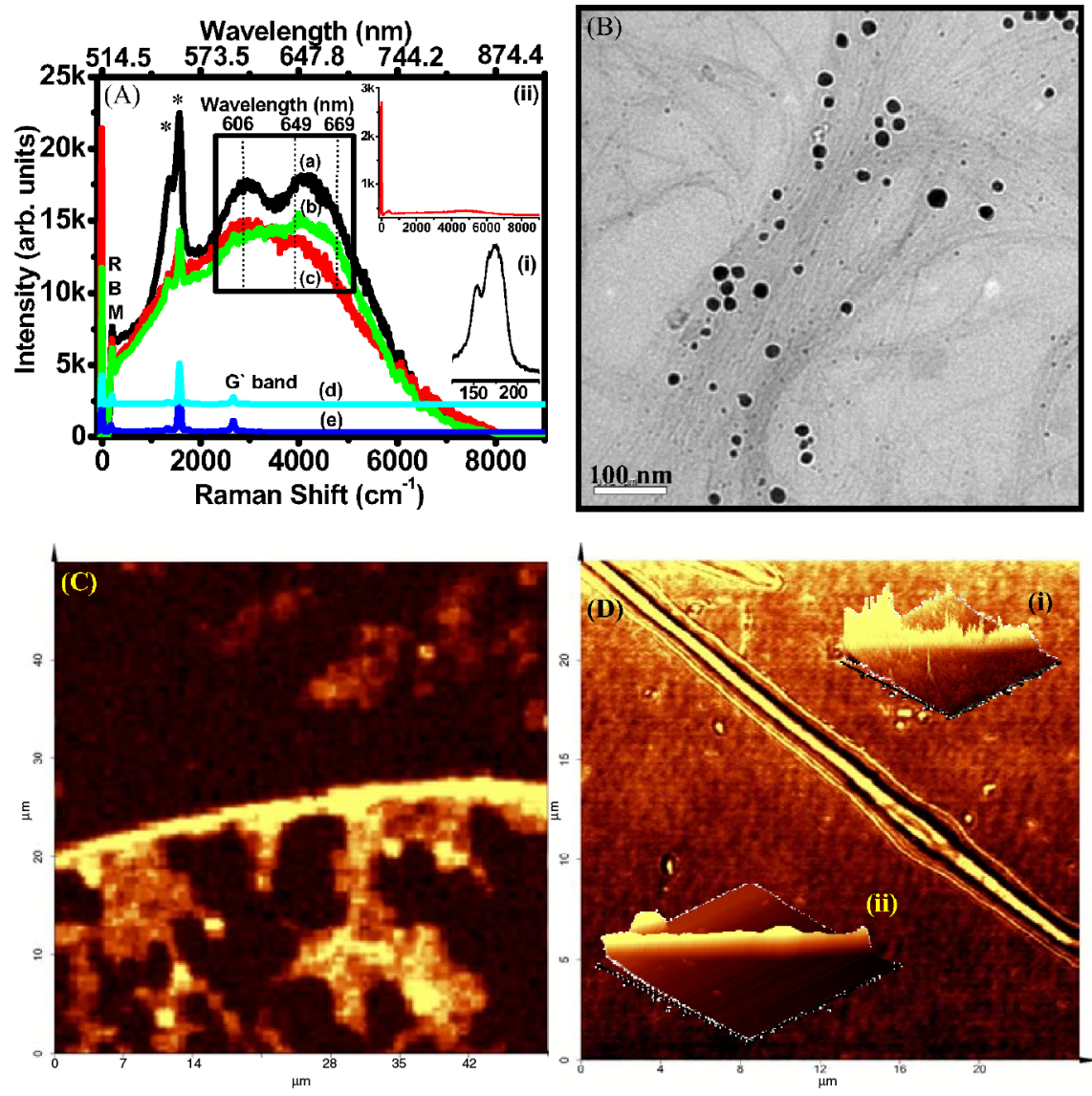

FIG. 1 (color online). (A) Single spot Raman spectra of (a) Ag-SWNT, (b) AuSWNT, (c) AuNR-SWNT, (d) pristine SWNTs, and (e) SWNTs treated with heated citrate solution. Traces (d) and (e) are shifted vertically for clarity. RBM is labeled and the $D$ and $G$ bands are indicated by asterisks $(*)$. The visible emission maxima are marked with their respective wavelengths. Inset (i): the $\mathrm{RBM}$ region at a higher resolution. Inset (ii): the Raman spectra of a dropcast film of AuNR, along with the Rayleigh to compare the intensities. (B) TEM image of the Au-SWNT. (C) Raman spectral images of AuNRSWNT, based on the intensities of visible emission in the 595 to $675 \mathrm{~nm}$ window. The spots observed away from the nanotube structure are due to shorter nanotubes remaining in the centrifugate or due to the $z$-axis discrimination of confocal imaging. (D) Light intensity based transmission SNOM image of AuNRSWNT. Inset (i): the three-dimensional view of (B), rotated suitably to show the increased emission. Inset (ii): corresponding topographic image. Raman and SNOM data are acquired with $514.5 \mathrm{~nm}$ excitation.

$167404-2$ 
(ii) of Fig. 1(A)]. AuNRs show a feature at $5000 \mathrm{~cm}^{-1}$, attributable to their weak photoluminescence [13] with quantum yield as low as $10^{-5}$. Further, Ag nanoparticle synthesized from vapor condensation method show similar emission features, implying that the route of nanoparticle synthesis does not influence the emission. It also rules out the role of the possible chemical impurities, during the synthesis, in the emission observed. Studies were conducted using a Nd: YAG $532 \mathrm{~nm}$ excitation to investigate the photophysical nature of the emission. Emission features at $\sim 600$ and $650 \mathrm{~nm}$, with a shoulder at $675 \mathrm{~nm}$, along with other Raman modes were observed. As emission wavelengths are independent of excitation wavelength, we classify them as fluorescence.

The composite was mapped using the intense Raman features and the visible fluorescence. A $50 \times 50 \mu \mathrm{m}$ image was constructed using the emission intensity between the 595 and $675 \mathrm{~nm}$ [Fig. 1(C)]. Similarity of the images due to RBM, $D$ and $G$ bands, and the visible fluorescence suggest that the emission is from SWNTs in the composite. The full width at half maximum (FWHM) of the image along the cross section is $1.78 \mu \mathrm{m}$, far above the classical optical resolution limit. The fluorescence features had small shifts $\left(\sim 20 \mathrm{~cm}^{-1}\right)$ along the length of the tube. Scanning near-field optical microscopy (SNOM) imaging, difficult on pristine SWNTs due to poor signals, has been successfully demonstrated using the intense visible fluorescence from the AuNR-SWNT composite [Fig. 1(D)]. The transmitted light intensity is greater than the background transmission [inset (i), Fig. 1(D)]. The FWHM of the cross section is $180 \mathrm{~nm}$ below the Abbe diffraction limit of $285 \mathrm{~nm}(\lambda=514.5 \mathrm{~nm}$, Numerical Aperture = $0.9)$. As the detector is sensitive only up to $800 \mathrm{~nm}$, the contribution from possible infrared emission of pristine SWNTs is unlikely. The topography [inset (ii)], taken along with the transmission images, rules out scanning induced artifacts. Similar Raman and SNOM images were collected from other composites.

We find a strong correlation between the $D$ band and the fluorescence intensities. Figure 2(a) shows that both the $D$ band and the visible fluorescence intensities decrease with $C_{\mathrm{NP}}$, for a fixed $C_{\mathrm{SWNT}}$. This indicates that nanoparticles induce defects on the nanotubes which render the latter fluorescent in the visible range. However, the properties of nanoparticles, namely, the surface plasmon absorption and the $\mathrm{Au} 4 f_{7 / 2} / \mathrm{Ag} 3 d_{5 / 2}$ binding energies, do not change. Further, the fluorescence intensity decreases with $C_{\text {SwNT }}$ for a given $C_{\mathrm{NP}}$ [Fig. 2(a), inset]. Moreover, the fluorescence is strongly sensitive to helium which is known to get selectively adsorbed on SWNTs [Fig. 2(b)]. However, no such sensitivity is found for nitrogen or air which does not interact with SWNTs [14]. All these confirm that the nanotubes in the composites indeed fluoresce.

We observe that the fluorescence disappears when either of the components of the composite are protected with CTAB for $C_{\text {СТАВ }}$ above $10^{-4} \mathrm{M}$, the latter being known to screen electrostatic interactions. TEM suggests that SWNTs does not form a composite at such $C_{\mathrm{CTAB}}$, the nanoparticles and nanotubes being phase separated [Fig. 2(b) inset]. Further, monolayer protected nanoparticles failed to show emission. Thus the composite formation proceeds predominantly via direct electrostatic interactions between the nanoparticles and SWNTs.

We further compare the transport properties of the composite and pristine SWNTs, for the defects are known to modify the electronic band structures of SWNTs. To this end, mSWNTs were extracted from HiPco synthesized SWNT bundles following a standard protocol [15], diluted and drop cast to have self-assembled, well-separated mSWNT bundles with typically 3 mSWNTs. Figure 3(a) shows the Raman spectra of pristine HiPco synthesized nanotubes (black trace) and mSWNTs extracted from them (red trace). The RBMs at $199 \mathrm{~cm}^{-1}$ and $277 \mathrm{~cm}^{1}$ are due to the semiconducting and metallic SWNTs, respectively, present in the HiPco synthesized sample. Note that the HiPco samples have different diameters and their RBMs are different [compare with Fig. 1(A)]. The RBM was used to assess the efficiency of extraction of mSWNTs from pristine SWNT [16,17]. The area under peaks at $199\left(A_{S}\right)$ and $277 \mathrm{~cm}^{-1}\left(A_{M}\right)$ were calculated which suggests that the percentage of mSWNTs in the sample is around $88 \%$.

Both mSWNTs and Au-mSWNT samples were subject to the point-contact current-imaging atomic force microscopy (PCI-AFM) [18,19]. A plot of conductance versus bias voltage for isolated nanotube structures in mSWNT and Au-mSWNT is presented Fig. 3(b). Zero conductance at zero voltage for the Au-mSWNT is in stark contrast to the metallic behavior of mSWNTs. This variation of elec-
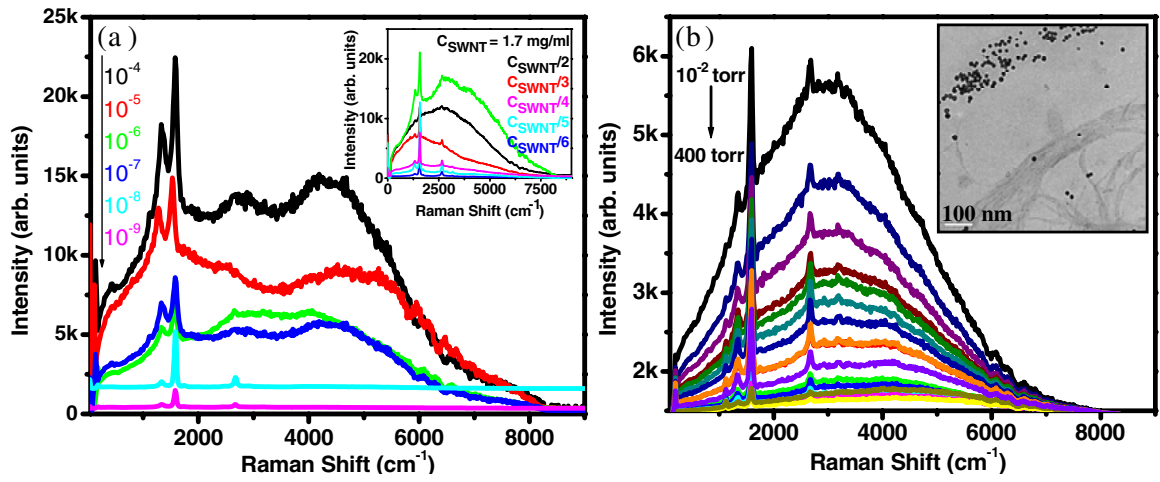

$167404-3$
FIG. 2 (color online). (a) Raman spectra of Ag-SWNT as a function of varying $C_{\mathrm{NP}}$ and $C_{\mathrm{SWNT}}$ (inset). (b) Fluorescence intensity variation from $\mathrm{Au}-\mathrm{SWNT}$ as a function of helium pressure. The highest intensity trace is in the absence of helium at a vacuum of $10^{-2}$ torr. Inset: TEM of $\mathrm{Au}-\mathrm{SWNT}$ at $C_{\mathrm{CTAB}} \sim 10^{-4} \mathrm{M}$, showing a clear separation between nanoparticles and SWNTs. 

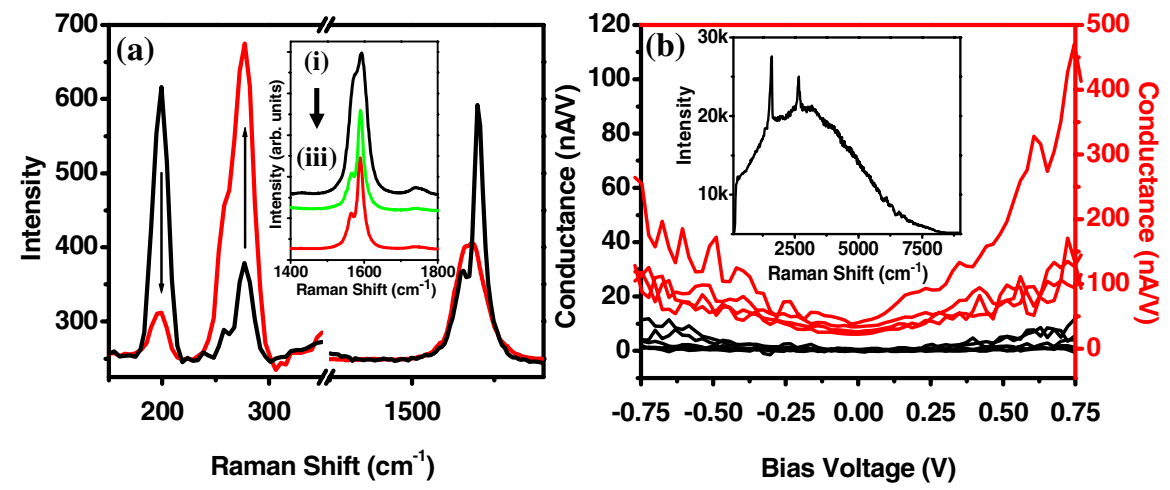

FIG. 3 (color online). (a) Raman spectra of pristine HiPco synthesized SWNTs (black trace) and extracted mSWNTs (red trace), in the RBM and $G$-band regions. Inset: Raman spectra of (i) extracted mSWNTs, (ii) Au-mSWNT, and (iii) pristine SWNTs in the $G$-band region. Note distinct change in FWHM with Au-mSWNT resembling pristine SWNTs. (b) Differential conductance of mSWNTs (red traces) and Au-mSWNT (black traces). Inset: Raman spectrum of Au-mSWNT, showing visible emission. trical transport properties [20] was seen consistently in all the samples analyzed, showing a metal-semiconductor (MS) transition in nanotubes. Since mSWNT bundles are metallic, the nanoparticle binding, not the intertube interaction in the bundle, results in the M-S transition. The $G$-band line shape is different for metallic and semiconducting SWNTs [21] with differences in the full width at half maximum (FWHM). The $G$-band region was monitored at various points of mSWNT and Au-mSWNT. Large reduction in FWHM [inset, Fig. 3(a)] confirms the changes in the electronic properties of the nanotubes [21].

The Raman spectrum on the Au-mSWNT shows the visible fluorescence [inset, Fig. 3(b)], qualitatively similar to those obtained from Au-SWNT, Ag-SWNT, and AuNRSWNT. The M-S transition in nanotubes brought by the nanoparticle/nanorods binding eliminates the nonradiative decay channels, thereby facilitating the visible fluorescence. The $E_{11}^{M}$ and $E_{23}^{S} / E_{32}^{S}$ interband transition energies for tubes of indices $(10,10),(18,0),(13,7),(17,0),(11,9)$, and $(12,8)$ are around $2 \mathrm{eV}$ [22] close to the observed fluorescence positions of $\sim 1.9$ and $\sim 2.1 \mathrm{eV}$.

The optical response of the localized electrons in the composite can be understood by simple classical arguments. Let us consider a one-dimensional chain, each bead having mass $M$, except that, at the center the mass $M^{\prime}$, represents a nanoparticle bound core. The nanoparticle bound core will be slowed down by a factor of $M / M^{\prime}$ due to the localized phonon vibrations [23], which can be treated fixed. The equation of motion of the electron, within harmonic approximation, shows that the frequency $\omega$ dependent induced dipole moment, $d_{\omega}$ has a pole at $\sqrt{2 V_{0}^{\prime \prime} / m}$ in the optical range, the curvature $V_{0}^{\prime \prime}$ being curvature of the core-electron interaction. The coreelectron interaction at a separation of $r$ has a short-ranged repulsion $\alpha(\sigma / r)^{12}$ and the long-ranged Coulomb attraction $-q e^{2} / \varepsilon r$. Here, $\sigma$ is the core diameter, $\alpha \sim k_{B} T\left(k_{B}\right.$ being the Boltzmann constant and $T$ the temperature), $q$ is the charge of the core, and $\varepsilon$ the dielectric constant of the medium. The dipole radiation, proportional to the square of $d_{\omega}$, will have diverging intensity in the optical range, consistent with the visible fluorescence.

In summary, interaction between the nanotubes and the nanoparticles destroys the metallicity of the nanotubes, facilitating the observation of visible emission from SWNT. The visible emission and concomitant metalsemiconductor transition will have immediate applications in sensing and biological imaging, with far reaching implications in the design and fabrication of nanoelectronic devices.

DST is thanked for financial support. C.S. acknowledges financial support from CSIR. Dr. K. George Thomas is thanked for AuNR samples used in the initial stages.

*pradeep@iitm.ac.in

[1] M. J. O'Connell et al., Science 297, 593 (2002).

[2] L. Huang, H. N. Pedrosa, and T.D. Krauss, Phys. Rev. Lett. 93, 017403 (2004).

[3] P. Cherukuri et al., J. Am. Chem. Soc. 126, 15638 (2004).

[4] N. W. ShiKam, M. O'Connell, J. A. Wisdom, and H. Dai, Proc. Natl. Acad. Sci. U.S.A. 102, 11600 (2005).

[5] T. Hertel, R. Fasel, and G. Moos, Appl. Phys. A 75, 449 (2002).

[6] J. S. Lauret et al., Phys. Rev. Lett. 90, 057404 (2003).

[7] A. Hartschuh et al., Chem. Phys. Chem. 6, 577 (2005).

[8] J. Turkevich, P. L. Stevenson, and J. Hiller, Discuss. Faraday Soc. 11, 55 (1951).

[9] N. R. Jana, L. Gearheart, and C. J. Murphy, Langmuir 17, $6782(2001)$

[10] F. Kim, J. H. Song, and P. Yang, J. Am. Chem. Soc. 124, 14316 (2002).

[11] T. K. Sau and C. J. Murphy, Langmuir 20, 6414 (2004).

[12] A. M. Rao et al., Science 275, 187 (1997).

[13] M. R. Beversluis, A. Bouhelier, and L. Novotny, Phys. Rev. B 68, 115433 (2003).

[14] C. Subramaniam et al. (unpublished).

[15] Y. Maeda et al., J. Am. Chem. Soc. 127, 10287 (2005).

[16] M.S. Dresselhaus et al., Carbon 40, 2043 (2002).

[17] R. Krupe et al., Science 301, 344 (2003).

[18] Y. Otsuka et al., Jpn. J. Appl. Phys. 41, L472 (2002).

[19] H. Tanaka et al., Adv. Mater. 18, 1411 (2006).

[20] A. Vijayaraghavan et al., Nano Lett. 5, 1575 (2005).

[21] A. Jorio et al., Phys. Rev. B 66, 115411 (2002).

[22] J. C. Charlier and Ph. Lambin, Phys. Rev. B 57, R15037 (1998).

[23] C. Kittel, Introduction to Solid State Physics (Wiley Eastern Pvt. Ltd., New Delhi, 1971), 3rd ed. 